\title{
Erratum to: Percutaneous Endoscopic Gastrostomy Tube Placement: A Single Center Experience
}

\author{
Ankur Gupta $\quad$ Anil K. Singh ${ }^{2}$ Deepak Goel ${ }^{3}$ \\ ${ }^{1}$ Department of Gastroenterology, Max Hospital, Dehradun, \\ Uttarakhand, India \\ ${ }^{2}$ Department of Neurosurgery, Max Hospital, Dehradun, \\ Uttarakhand, India \\ ${ }^{3}$ Department of Neurology, Max Hospital, Dehradun, Uttarakhand, \\ India \\ ${ }^{4}$ Department of Oncosurgery, Max Hospital, Dehradun, \\ Uttarakhand, India \\ ${ }^{5}$ Department of Radiology, Max Hospital, Dehradun, Uttarakhand
} Akash N. Gaind ${ }^{4}$ Shireesh Mittal ${ }^{5}$ Address for correspondence Dr. Ankur Gupta, Department of
Gastroenterology, Sahara Hospital, Viraj Khand, Gomti Nagar,
Lucknow 226010, India (e-mail: mail.guptaankur@gmail.com).

J Digest Endosc 2019;10:e1

\section{Erratum}

Authors have informed the Publisher that they wish to update affiiations 1-4 in the above-mentioned article, published eFirst on December 12, 2019. DOI of the original article is DOI: $10.1055 / \mathrm{s}-0039-3401391$.

Correct affiliations are as follows:

'Department of Gastroenterology, Max Hospital, Dehradun, Uttarakhand, India ${ }^{2}$ Department of Neurosurgery, Max Hospital, Dehradun, Uttarakhand, India ${ }^{3}$ Department of Neurology, Max Hospital, Dehradun, Uttarakhand, India ${ }^{4}$ Department of Oncosurgery, Max Hospital, Dehradun, Uttarakhand, India 